\title{
Las competencias laborales y el cumplimiento del rol de las secretarias
}

\section{Labor competencies and the fulfillment of the role of secretaries}

\section{Las competencias laborales}

Karen Yelena Cedeño León, estudiante. ${ }^{(1)}$

Reynier García Rodríguez, PhD. ${ }^{(2)}$

(1) Carrera de Secretariado Gerencial del Instituto Superior Tecnológico, Portoviejo. Ecuador. email: karenyelenac@gmail.com ORCID: https://orcid.org/0000-0001-5907-028X

(2) Instituto Superior Tecnológico, Portoviejo, Ecuador. email: reygarod79@ gmail.com ORCID: https://orcid.org/0000-0002-4160-5749

Contacto: karenyelenac@gmail.com

Recibido: 18-02-2020 Aprobado: 06-04-2020

\section{Resumen}

La investigación se basa en las competencias laborales como configuración psicológica, donde se aprecia desde el contexto empresarial ecuatoriano como la integración de cuatro componentes fundamentales: cognitivo, motivacional -afectivo, metacognitivo y funcional; donde el centro de la competencia en la motivación; resulta único y distintivo, que es la primera vez en Ecuador que se hace un análisis de tal connotación en el ámbito empresarial. El presente artículo tiene como objetivo analizar las competencias laborales del secretario, a partir de su determinación como configuración psicológica y la medición en la práctica de sus elementos distintivos en las secretarias del cantón 24 de mayo de la provincia de Manabí. Se emplearon métodos del orden teórico, análisis de síntesis, inductivo - deductivo e histórico lógico; los que permitieron incursionar en la teoría y en la práctica. También se utilizaron métodos del orden empírico, como la encuesta dirigida a un total de 25 colaboradores que ejercen su cargo como secretarias. Se obtuvo como resultado, la configuración de la competencia, valorándose de manera preliminar en la práctica y su relación con el desempeño profesional. Se concluye que en la investigación se aprecian las competencias laborales del secretario y el rol en su desempeño profesional, así como la concreción de los resultados profesionales, empresariales y contextuales: económico, social y cultural de la empresa. Lo que consecuentemente repercute en el ambiente institucional, el grado de satisfacción y motivación de los trabajadores, en favorables relaciones interpersonales e imagen positiva de la empresa, comunidad y contexto laboral.

Palabras clave: competencias laborales, configuración psicológica, desempeño profesional
The research is based on work skills as a psychological configuration, where it is appreciated from the Ecuadorian business context as the integration of four fundamental components: cognitive, motivational-affective, metacognitive and functional; where the center of competition in motivation; It is unique and distinctive, which is the first time in Ecuador that an analysis of such connotation has been made in the business world. The purpose of this article is to analyze the secretary's labor competencies, based on his determination as a psychological configuration and the measurement in practice of its distinctive elements in the secretaries of the May 24 canton of the province of Manabí. Methods of the theoretical order, analysis of synthesis, inductive-deductive and logical historical were used; those that allowed them to dabble in theory and practice. Empirical methods were also used, such as the survey directed at a total of 25 collaborators who are serving as secretaries. As a result, the configuration of the competition was obtained, being preliminarily valued in practice and its relationship with professional performance. It is concluded that the investigation shows the secretary's work competences and the role in his professional performance, as well as the concretion of the professional, business and contextual results: economic, social and cultural of the company. What consequently affects the institutional environment, the degree of satisfaction and motivation of workers, favorable interpersonal relationships and a positive image of the company, community and work context.

Keywords: occupational competence, psychological settings, professional performance

\section{Introducción}


A nivel internacional, la situación socioeconómica, política y educativa se complejiza y se evidencia de diversas formas. El vertiginoso y acelerado desarrollo de la ciencia y la tecnología invaden el ámbito empresarial; así como las demandas de empleo que se acrecientan cada vez y busca que los países atiendan a las potencialidades de las personas; como forma de supervivencia y desarrollo permanente.

Ecuador en este sentido, se suma a una constante transformación empresarial, que abarca a los directivos, secretarios, funcionarios, proveedores y toda la comunidad empresarial que gira en torno a la institución o entidad. Se trata de garantizar el bienestar del hombre con la intención de perfeccionarlo con una visión humanista, solidaria, distintiva y única.

Las ciencias empresariales y técnicas, poseen un rol definitivo en el mejoramiento permanente de los procesos formativos y desarrolladores del hombre actual; a través de su práctica social concreta y por ende, debe tributar como ciencia al desarrollo del país. El mejoramiento mencionado, está implícito en los procesos que se llevan a vías de efecto en las empresas ecuatorianas, las que deben abrirse a su contexto, generando retos permanentes de aprendizajes individuales y colectivos. (Cuik \& Pérez, 2018)

Por tal razón, la empresa ecuatoriana es un eje esencial en el desarrollo tecnológico actual y perspectivo del país, tiene como encargo social, la formación integral de los secretarios es demandada por la sociedad. Para este fin, es necesario desde las investigaciones tributar a la competencia profesional entendida como: la integración de conocimientos, habilidades, valores, motivaciones, emociones, actitudes, aptitudes y modos de desempeño profesional acorde al desarrollo del país (Guaña, Quinatoa \& Pérez 2017).

Para el cumplimiento de la misión, visión, políticas empresariales a nivel local, provincial, nacional e internacional; hay que prestar atención a la figura del secretario. En este sentido, vale destacar que en las empresas se evidencia un amplio diapasón en su objeto social, atendiendo a sus producciones y servicio; lo que influye a mediano y largo plazo en el alcance de la calidad empresarial y en los resultados del trabajo, a partir de un desempeño profesional exitoso.

Una mirada hacia el secretariado en Ecuador permite discurrir en que existen diversos estudios en torno a las competencias laborales, sin embargo, hay carencias de estos estudios en los profesionales que ejercen como secretarios. En otro orden, existen estudios en torno a competencias en diversas áreas del saber, sin embargo, hay carencias de estudios relacionados con la competencia profesional como configuración psicológica. Finalmente, no se evidencia una posible solución para el desarrollo de las competencias laborales del secretario ejecutivo.

Estas dificultades evidenciadas de manera empírica, permiten identificar una contradicción fundamental entre las exigencias que se plantean al rol del secretario ejecutivo y el insuficiente desarrollo de las competencias laborales.

Las derivaciones contradictorias anteriormente expresadas, permiten definir una interrogante: ¿A qué se le denomina competencias laborales del secretario ejecutivo? ¿Cuál es la concepción de las competencias laborales como configuración psicológica? ¿Cómo contribuir al desarrollo de las competencias laborales del secretario ejecutivo?

El trabajo tiene como objetivo, analizar las competencias laborales del secretario, a partir de su determinación como configuración psicológica y la medición en la práctica de sus elementos distintivos en las secretarias del cantón 24 de mayo de la provincia de Manabí.

\section{Materiales y métodos}

La investigación se realizó bajo los principios de la ética profesional; con criterios de responsabilidad, confidencialidad, consentimiento y devolución de los resultados a las instituciones donde pertenecen las 25 secretarias del cantón 24 de mayo, provincia de Manabí, Ecuador.

Se realizó un estudio descriptivo, prospectivo y transversal en 25 secretarias de diferentes instituciones del cantón 24 de mayo, en el periodo comprendido desde mayo de 2019 a abril de 2020.

En la investigación se tuvo en cuenta los secretarias de diversas instituciones; seleccionados de manera aleatoria a partir de las variables que se han delimitado de la siguiente manera: nivel de escolaridad, especialidad estudiada, elementos de la competencia, desempeño y capacitación; enfatizándose en los elementos de las competencias laborales y el rol que desempeñan.

Para el abordaje teórico - metodológico de las competencias laborales y el cumplimiento del rol de las secretarias, se partió de los objetivos enfocados al desarrollo de las competencias mencionadas y así mismo las modalidades de trabajo que coadyuvan a la capacitación de las secretarias según lo planteado por (García, 2015).

La figura 1 muestra la Metodología para el desarrollo de las competencias laborales 


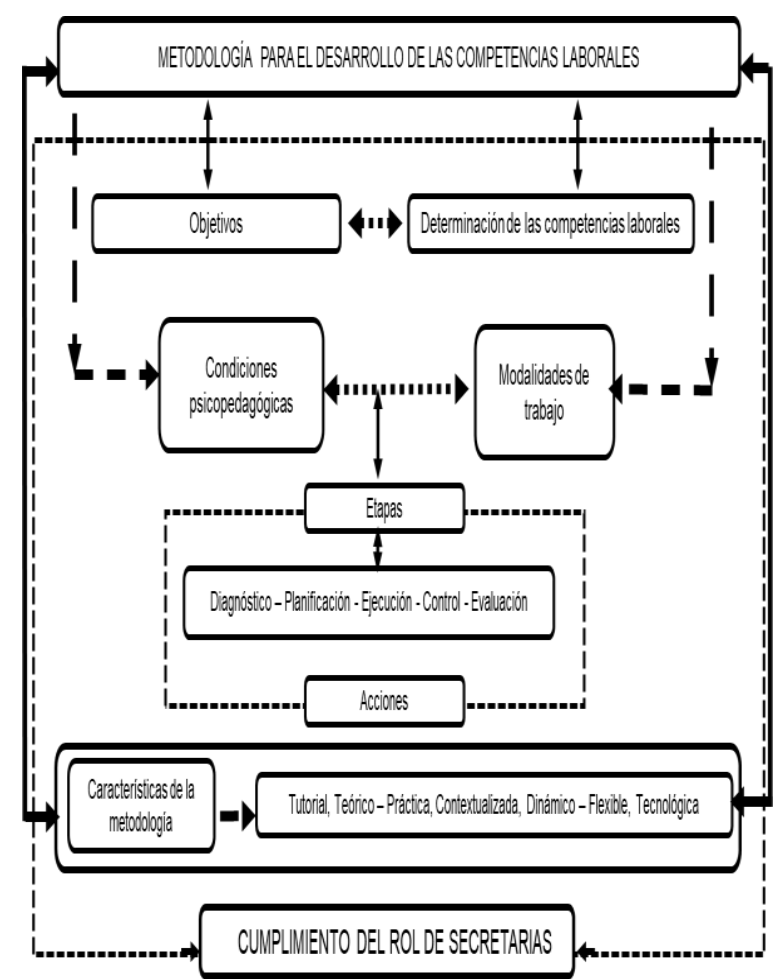

Figura 1. Metodología para el desarrollo de las competencias laborales

La determinación de las competencias se logró a partir de la sistematización de la teoría y la elaboración de instrumentos empíricos. En este sentido, logró la identificación de las condiciones psicopedagógicas de la metodología: participación protagónica, activa y consciente, establecimiento de normas, redimensión del rol del facilitador, contextualización, implementación de las TIC, observancia de las relaciones entre los componentes didácticos y aprovechamiento de la experiencia profesional. En este orden, en las modalidades de trabajo se logró la identificación de: curso de capacitación presencial y de educación a distancia, asesorías, orientaciones y talleres.

Se procedió al diseño de una base de datos para la realización del análisis estadístico inferencial, a partir del estudio del cálculo porcentual, empleándose el programa Microsoft Office Excel; donde se consideraron las frecuencias absolutas y relativas.

\section{Resultados}

En el periodo comprendido desde mayo de 2019 a abril de 2020, se logró la concreción de la investigación a partir del estudio de las 25 secretarias. Vale destacar que 11 son bachilleres en diversas áreas (44\%) y 14 poseen títulos del tercer nivel $(56 \%)$ pero ninguno en secretariado o al menos relacionados con el rol que desempeñan.

Una vez que se logró la determinación de las competencias laborales, 20 secretarias (80\%) concordaron en que en una organización se evalúan las competencias laborales de las secretarias: capacidades, habilidades y aptitudes de un profesional; sin embargo no se tiene en cuenta las motivaciones, metacogniciones, ni componentes funcionales de la personalidad. El resto de las secretarias, plantean que solo cumplen con sus funciones según reciben orienctaciones a diario, semanal o mensual.

Relacionado con el rol profesional, 16 de las secretarias (64\%) plantean que el clima favorable de las empresas dependen de la organización que una secretaria pueda garantizar; en cambio 9 de ellas (36\%) no logran apreciar la relación entre el rol profesional y el clima de la empresa.

En cuanto a la capacitación, una secretaria (4\%) reveló que domina las competencias laborales relacionadas con conocimientos, habilidades y destrezas. En tanto, 24 secretarias (96\%) revelaron que carecen de conocimientos, habilidades $\mathrm{y}$ destrezas; además de insuficiencias en la identificación del componente funcional de la personalidad ni la valía de la metacognición. Una de las dificultades que se evidencia es que no participan en la toma de decisiones de la institución a la que pertenecen.

Se develó además que, las competencias laborales influyen en el rendimiento de las instituciones, según el criterio de 12 secretarias (48\%), en tanto 13 secretarias $(52 \%)$ plantearon que las competencias además de relacionarse con el rendimiento, solo se pueden apreciar en el rol que desempeñan, mostrando así: conocimientos, habilidades, motivaciones, metaconocimientos, asertividad, flexibilidad y valores éticos, estéticos y morales.

Finalmente, las competencias laborales estuvieron con deficiencias en el $100 \%$ de las secretarias encuestadas.

\section{Discusión}

El término competencia es antiguo y su apreciación más fecunda se apreció, entre los años 1970 - 1979; destacándose McClelland (1973) y luego, Gonzci (1996); Mertens (1996); Ducci; Gallard (1997); Agudelo (1998); Le Botert (1998); Kochanski (1998); Zarifian (2001); Miranda (2003); Fernández (1997); Romeu (1997); Rodríguez (2002); Becerra (2003); Sarmiento (2004); Martínez (2004); Santos (2005); Cejas (2005); Trocones (2005); Pelegrín (2006); Pérez (2006); Alpízar (2007); Rodríguez (2009), García (2014,2019). Es un término que amigable que se ha introducido en todas las áreas y profesiones.

Aun cuando es amigable, no deja de ser polémico y se debate con fuerza por su polisemia. Todo investigador debe dominar que coexisten dos grandes interpretaciones: competer (demostración de dominios, eficacia, eficiencia y se enfoca en el éxito de la persona) y competir (rivalidad entre pares, enfrentamiento). En el caso del secretariado como 
profesión, se relaciona con estrategias que generan ventajas competitivas, mayor nivel de productividad, empleabilidad y gestión del talento humano; que se traduce en desarrollo y calidad. (Díaz \& Viera, 2019)

En esta investigación se concuerda con los autores Díaz \& Viera (2019), quienes declararon que es un sistema de conocimientos, habilidades, valores y cualidades de la personalidad; una apropiación dialéctica de la realidad y que cada elemento es un instrumento que pondera la competitividad. También se coincide con otras investigaciones realizadas por Díaz \& Viera (2019), en la que hacen alusión a la competencia, con un enfoque configuracional desde los aspectos psicológicos. Luego de aplicar la metodología el $100 \%$ de las secretarias coincidieron en que el concepto es imposible de limitar, dada la variedad de elementos que lo componen.

En el intercambio con las secretarias, se llegó al consenso en el $95 \%$ que el enfoque de las competencias laborales es configuracional. En tanto, construyen en el proceso de interacción social y expresan la autodeterminación de la persona en el ejercicio eficiente y responsable de la profesión, se concuerda con (Mancebo \& Betancur, 2018).

El 100\% de las secretarias logró comprender y asumir que el éxito de su rol profesional se logra a través del desarrollo de las competencias profesionales, apreciándolas como una: "Configuración psicológica compleja que integra en su estructura y funcionamiento formaciones motivacionales, cognitivas y recursos personológicos que se manifiestan en la calidad de la actuación profesional del sujeto, y que garantizan un desempeño profesional responsable y eficiente, se coincide con (González, 2002).

Del total, 24 secretarias $(96 \%)$ coinciden con que las competencias profesionales, además de ser una configuración psicológica; plantean que autorregulan el desempeño real y eficiente del profesional, en su área de acción. Por su parte, una secretaria (4\%) indicó que asume los aportes del resto añadiendo que el eje de las competencias es la motivación.

El $100 \%$ de las secretarias planteó que las competencias laborales como configuración psicológica, se interrelacionan con los componentes distintivos que las integras: cognitivo, metacognitivo, motivacional - afectivo y funcional.

La posibilidad brindada por la investigación, permitió que el $100 \%$ de las secretarias participaran en la operacionalización de las competencias profesionales, obteniéndose como aspectos relevantes que: el componente cognitivo contiene un sistema de conocimientos (saberes y habilidades), que se calza por los procesos y propiedades del intelecto: sensación, percepción, memoria, imaginación y el pensamiento; constituyendo una unidad. En el secretario debe revelarse, la independencia en la logicidad del pensamiento, la originalidad traducida en la variedad de ideas, la fluidez en la generación de las ideas con su consabida contextualización, la profundidad que permite analizar hechos y fenómenos y, la economía de recursos en la consecución de tareas).

Desde lo cognitivo, el secretario debe poseer una base de conocimientos que le permita la operación del ejercicio de su profesión relacionada con el contexto donde se desempeña, en este sentido: conocimientos legales, axiológicos, investigativos, protocolares, tecnológicos, psicológicos, procedimentales, actitudinales. (Díaz \& Viera, 2019). También depende de las habilidades, las que constituyen el dominio de acciones prácticas que garantizan la regulación racional de las tareas inherentes a su rol.

Las habilidades se dirigen a:

- Proyectar estratégicamente la empresa, colaborando con las autoridades en pos del objeto social del que se trate.

- Aplicar técnicas de dirección en cuanto a la valoración del recurso tiempo, la adecuada planificación de las actividades, propendiendo a la asistencia, puntualidad, aprovechamiento de la jornada laboral y a la producción y servicios.

- Gestionar las transformaciones de la empresa, en cuanto a la actualización de su modelo económico a nivel local, regional, nacional e internacional. Implica comunicar los cambios y los resultados, planificar las acciones, proyectar metas y objetivos.

- Garantizar una comunicación afectiva y efectiva, implica la comunicación dentro y fuera de la empresa, ponderando el logro de la relación eficaz a través de una buena escucha, observación y relación empática.

- Incidir en la cooperación inter empresarial. En este sentido, la atención a los cambios tecnológicos, las demandas del sector productivo, el comportamiento de la economía en su vínculo con demandas sociales.

El componente metacognitivo, organiza y planifica la actividad cognoscitiva, lo que se concreta en determinar los objetivos y las estrategias cognitivas a emplear, planificar el desarrollo de la actuación y controlar la acción realizada.

La reflexión metacognitiva se refiere a la percepción del secretario en relación con sus conocimientos y además valora con objetividad su propio desempeño 
y tiene conocimientos de sus habilidades, accionar e interactuar en la empresa.

La regulación metacognitiva se refiere a la evaluación y corrección de la calidad en su desempeño profesional. Supervisa la calidad de la ejecución de su accionar en función del desarrollo de los recursos personológicos.

El componente motivacional puede entenderse a partir de considerar el motivo "como elemento que estimula y promueve las acciones del hombre con vista a satisfacer sus necesidades. El componente motivacional está en el centro de las competencias laborales como una configuración. Se coincide con Turienzo, (2016), en este orden, motivación por la profesión, motivación por la empresa y valores asociados: compromiso, responsabilidad en la ejecución de funciones y tareas; así como la laboriosidad y ética profesional.

Sobre la base de lo anterior, el componente funcional es un recurso que moviliza en función del desempeño profesional, que implica el reconocimiento y dominio del carácter y el temperamento, el control emocional y la actitud para la toma de decisiones. Se concuerda con García, (2019), en la que reflexiona sobre el autocontrol emocional, asertividad y flexibilidad; centrando su quehacer en el cumplimiento de la misión, visión y políticas institucionales.

Los componentes de las competencias laborales se desarrollan a través de la metodología propuesta, donde se tiene en cuenta las etapas: diagnóstico, planificación, ejecución, control y evaluación; con acciones concretas y precisas en torno al rol de las secretarias.

En 24 secretarias (96\%) se evidenció el desarrollo de los componentes de las competencias laborales y la secretaria restante (4\%) un acercamiento al desarrollo de la competencia, donde se precisa profundizar en los componentes funcionales de la personalidad.

\section{Conclusión}

Se concluye que en la investigación se aprecian las competencias laborales del secretario y el rol en su desempeño profesional, entendido como la concreción de los resultados profesionales, empresariales y contextuales: económico, social y cultural de la empresa. Lo que consecuentemente repercute en el ambiente institucional, el grado de satisfacción y motivación de los trabajadores, en favorables relaciones interpersonales e imagen positiva de la empresa, comunidad y contexto laboral. Finalmente, el desarrollo de las competencias laborales debe ser de manera permanente y sistemática, propiciándose el realce de sus componentes.

\section{Bibliografías}

Cuik, A. A., \& Viera, O.. (2018). La comunicación en el Sistema Organizativo de Dirección.

Díaz, N. P., \& Viera, O. P. (2019). La comunicación organizacional en las instituciones.

García, R. R. (2019) Apuntes sobre la evaluación, la neurociencia y la autoevaluación en la formación de profesionales. REEA 1, (4). Recuperado de http://www.eumed.net/rev/reea/agosto19/evaluacion-docencia.html

Guaña,E., Quinatoa, E \& Pérez, M. (2017). Tendencias del uso de las tecnologías y conducta del consumidor tecnológico. Revista Ciencias Holguín, 23, (2). Recuperado de https://www.redalyc.org/pdf/1815/18155095 9002.pdf

González, V. (2006). La formación de competencias profesionales en la universidad: reflexiones y experiencias desde una perspectiva educativa. Recuperado de https://core.ac.uk/download/pdf/41563302.pd $\mathrm{f}$

Portuondo, L. \& Jardínes, R. (2020). Tareas docentes para contribuir a la competencia profesional

de resolución de problemas de cálculo químico cuantitativo en la Educación de Adultos. Opuntia Brava, 12(1), 21-36. Recuperado de http://opuntiabrava.ult.edu.cu/index.php/op untiabrava/article/view/950/1121

Ricardo, A., Figueredo, P., \& Pupo, M. (2019). La estrategia para la orientación profesional en las carreras empresariales universitarias: su aplicación e impacto. Rev. Opuntia Brava, 11(2), 90-106.

Turienzo, R. (2016). El pequeño libro de la motivación. Ed. Alienta. Recuperado de https://www.planetadelibros.com/libros_cont enido_extra/34/33578_EL_PEQUENO_LIB RO_DE_LA_MOTIVACION.pdf

Mancebo, M. E., \& Bentancur, N. (2018). Las competencias en la educación superior: nudos críticos y oportunidades de innovación. Inter Cambios. Dilemas y transiciones de la Educación Superior, 5(1),104-115.

Ortega, F. (2011). Ideario de Simón Rodríguez. Moral y luces. Rev. de Estudios Sociales,(38) Recuperado 
http://journals.openedition.org/revestudsoc/1 1451

Vargas, I. R., Payrol, J. A., \& Quijano, M. M. (2019). El enfoque de procesos, su concreción en un manual de gestión institucional. Revista Boletín Redipe, 8(3), 125-134. 